Résumés des conférences et travaux

\title{
Grammaire comparée des langues indo-européennes
}

\section{Linguistique et philologie baltiques}

Daniel Petit

\section{(2) OpenEdition}

Journals

Édition électronique

URL : https://journals.openedition.org/ashp/389

DOI : $10.4000 /$ ashp.389

ISSN : 1969-6310

Éditeur

Publications de l'École Pratique des Hautes Études

\section{Édition imprimée}

Date de publication : 1 octobre 2008

Pagination : 351-353

ISSN : 0766-0677

\section{Référence électronique}

Daniel Petit, "Linguistique et philologie baltiques », Annuaire de l'École pratique des hautes études (EPHE), Section des sciences historiques et philologiques [En ligne], 139 | 2008, mis en ligne le 26 novembre 2008, consulté le 12 juillet 2021. URL : http://journals.openedition.org/ashp/389 ; DOI https://doi.org/10.4000/ashp.389 


\title{
LINGUISTIQUE ET PHILOLOGIE BALTIQUES
}

\author{
Chargé de conférences : M. Daniel PetiT
}

Programme de l'année 2006-2007 : I. Introduction au vieux prussien; - II. Lecture linguistique d'extraits du III catéchisme prussien (1561).

L'objet du cours de cette année a été une introduction à la langue et à la philologie du vieux prussien. Il visait avant tout à remplir une lacune dans les études linguistiques, puisque c'est la première fois qu'un cours portant sur cette langue baltique archaïque a fait l'objet d'un enseignement universitaire en France. Or le vieux prussien n'est pas sans intérêt pour la linguistique comparative indo-européenne, ni bien évidemment pour l'histoire culturelle et linguistique des territoires de la Baltique orientale.

À l'intérieur de la famille des langues baltiques, la position du vieux prussien est relativement précaire. Il s'agit de la seule langue baltique occidentale attestée par des documents écrits et, à ce titre, le vieux prussien ne peut être comparé qu'indirectement au baltique oriental, représenté par le lituanien et le letton, avec lesquels il présente plusieurs divergences profondes. Les documents prussiens sont, en outre, limités à la fois en nombre et en extension; on peut mentionner les sources suivantes :

(1) L'« épigramme de Bäle », découverte en 1974 par Stephen C. Mc Cluskey et qui pourrait dater de la seconde moitié du XIV siècle; le texte ne laisse aucun doute sur le fait qu'il s'agit d'une chanson à boire, mais le détail de l'analyse reste obscur :

Kayle rekyse. thoneaw labonache thewelyse.

Eg. koyte. poyte nykoyte. pênega doyte.

(traduction approximative :

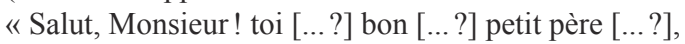

si tu veux boire, tu ne veux pas donner d'argent »)

(2) Le « vocabulaire d'Elbing » (en abrégé : EV), un vocabulaire allemand-prussien d'environ 800 items, daté des environs de 1400, mais qui doit être la copie d'un texte écrit au début du XIV ${ }^{\mathrm{e}}$ siècle ou à la fin du XIII ${ }^{\mathrm{e}}$ siècle.

(3) Le « vocabulaire de Simon Grunau » (en abrégé Gr), un vocabulaire d'une centaine de mots prussiens et allemands, transmis par l'auteur dans sa Preussische Chro$n i k(1517-1526)$.

(4) Trois catéchismes luthériens (Cat. I, II, III) publiés à Königsberg, le premier et le second en 1545 , le troisième en 1561.

Le vieux prussien, parlé autrefois en Prusse orientale dans la région de Königsberg (l'actuelle Kaliningrad), est sorti de l'usage aux alentours de 1700. Après cette date, les langues parlées dans cette région ont été le lituanien (c'est d'ailleurs le dialecte de Prusse orientale qui a fourni la base de la langue lituanienne standard) et l'allemand; 
depuis la fin de la seconde guerre mondiale, cette région, très dévastée d'un point de vue culturel, est occupée par des populations russophones.

Durant le cours, on a proposé une introduction à trois des aspects les plus caractéristiques du vieux prussien : (1) la diversité dialectale, et notamment les différences qui séparent le vocabulaire d'Elbing et les catéchismes, en particulier d'un point de vue phonétique; (2) la morphologie nominale, pronominale et verbale, envisagées à travers le prisme de l'influence allemande sur la langue et/ou les textes prussiens; (3) la syntaxe, éminemment soumise à la pression de l'allemand.

Sur le premier point, on peut résumer les acquis comme suit. Il est admis que le vocabulaire d'Elbing a été rédigé dans la région d'Elbing (aujourd'hui Elbłąg au Nord-Est de la Pologne), tandis que les catéchismes ont vu le jour dans la région de Königsberg. Le traducteur du III e catéchisme (1561), Abel Will, était prêtre à Pobethen (aujourd'hui Romanovo, dans la région autonome de Kaliningrad), à environ $25 \mathrm{~km}$ au nord-ouest de Königsberg. On a donc des raisons que le vocabulaire d'Elbing relève d'un dialecte prussien occidental ou « poméranien », les catéchismes d'un dialecte prussien oriental ou « sambien ». Entre les deux sources il existe un grand nombre de différences, qui ont été discutées durant le cours, par exemple :

- la voyelle baltique *é était ouverte en poméranien, d'où des graphies comme 〈e〉, 〈ee〉, 〈ea〉 ou 〈ey〉 dans EV (par ex. semen « semence » EV $256<*$ sèmen-, wosee « chèvre » EV $676<* \bar{a} z \bar{e}$, geasnis « bécasse » EV $753<*$ gēsnis, seyr « cœur» EV $124<* \hat{k} \bar{e} r$ ). La même voyelle apparaît fermée en sambien (probablement */ẹ/), d'où une graphie 〈e〉 dans le Ir catéchisme et une évolution vers $* \bar{i}$, noté $\langle i\rangle,\langle\overline{1}\rangle,\langle\mathrm{y}\rangle$ ou $\langle\mathrm{ij}\rangle$ dans les II ${ }^{\mathrm{e}}$ et III ${ }^{\mathrm{e}}$ catéchismes (par ex. turrettwey « avoir » I $5_{3-4}<*$ turē-t- en regard de turrītwey III 27 7 ).

- la voyelle baltique $* \bar{a}$ était arrondie en poméranien et se confondait avec l'ancien * o (par ex. brote « frère » EV $173<*$ brāte, mothe « mère » EV $170<*$ mātēe, comme podalis «pot» EV $351<*$ pōd-elis). La même voyelle *ā était conservée comme telle en sambien (par ex. brāti « frère » III 67 ${ }_{10}$ ), mais passait à * $\bar{u}$ après labiale (par ex. $m \bar{u} t i$ « mère » III $67_{3}$ ). Le traitement de $* \bar{o}$ en sambien a fait l'objet d'une discussion détaillée durant le cours; il reste peu clair (conservation $\langle\overline{0}\rangle$ ? évolution vers $\langle\bar{a}\rangle$ ou $\langle\overline{\mathrm{u}}\rangle$ après labiale?).

- le sambien présente une tendance à diphtonguer les voyelles longues $*_{i}$ vers $\langle e \mathrm{i}\rangle$ ou 〈ej〉 et $* \bar{u}$ vers $\langle\mathrm{ou}\rangle \mathrm{ou}\langle\mathrm{au}\rangle$ (par ex. geiwans « vivant», acc. pl. I $9_{5}$, geywans II $9_{5}$, geīwans III $127_{15-16}$ en regard de gijwans III $43_{7}<*$ givva- ou sounon « fils », acc. sg. II $7_{12}$, soünon III $41_{22}$, saūnan III $123_{33}<*$ sünu-).

Le second aspect développé durant le cours a été la morphologie, pour laquelle on s'est appuyé principalement sur la langue des catéchismes, dans la mesure où EV et $\mathrm{Gr}$ ne présentent que des listes de mots. La difficulté principale à laquelle on est confronté quand on cherche à décrire la morphologie prussienne est celle de l'interprétation linguistique des graphies. Il est bien connu que les catéchismes ont été rédigés par des prêtres allemands, qui n'avaient sans doute qu'une connaissance sommaire, voire aucune connaissance, de la langue prussienne et se contentaient de recopier les formes dictées par des informateurs illettrés. Dans ces conditions on comprend les perturbations morphologiques observées dans les catéchismes. 
Pour la morphologie nominale, on a notamment évoqué longuement deux problèmes, tout d'abord le nombre des cas et la question du syncrétisme (il semble que le prussien ait réduit son système casuel à quatre cas sur le modèle de l'allemand, mais il existe certainement des vestiges de vocatif, et peut-être même de locatif et d'instrumental, quoique cela reste douteux) et surtout le problème bien connu des « constructions mixtes ", si caractéristiques du $\mathrm{III}^{\mathrm{e}}$ catéchisme. Par " construction mixte », on entend le fait que des formes associées peuvent apparaître à des cas différents : ainsi dans le groupe sen stesmu wirdan « avec le mot » (III 61 ${ }_{21}, 87_{22}$ ), l'article défini (luimême calqué sur l'allemand) est au datif (stesmu), tandis que le nom est à l'accusatif (wirdan). On considère en général que ces constructions ont été causées par l'interférence de l'original allemand (mit dem Wort), où l'article était clairement un datif (dem), tandis que le nom, en raison de la sous-spécification casuelle qui caractérise l'allemand, était ambigü (Wort peut être un accusatif ou un datif). Durant le cours, on a étudié plus en détail les conditions dans lesquelles apparaissent ces constructions mixtes et qui tiennent en général à la pression de l'allemand. Pour la morphologie pronominale, on a envisagé dans une approche comparable la flexion des pronoms personnels. Par exemple, les flottements au pluriel entre l'accusatif et le datif («nous » : acc. mans, dat. noumans, mais on trouve aussi mans comme datif et noumans comme accusatif) s'expliquent par l'indistinction formelle qui règne en allemand (uns, accusatif et datif). Au passage, on a discuté de l'évolution du supplétisme des pronoms personnels de l'indo-européen au baltique, à travers une comparaison du vieux prussien, du lituanien et du letton. Pour la morphologie verbale, enfin, les thèses novatrices de Wojciech Smoczyński (Lexikon der altpreussischen Verben, 2005) sur le système verbal ont été discutées et partiellement réfutées; on a notamment insisté sur la reconstruction du système modal (discussion sur l'impératif et le conditionnel en vieux prussien, dont l'existence et la formation ont été niées à tort par Smoczyński) et sur la classification des thèmes verbaux (élimination des doublets postulés inconsidérément par Smoczyński).

La syntaxe, enfin, a été largement évoquée lors des séances consacrées à la lecture linguistique d'un passage du $\mathrm{III}^{\mathrm{e}}$ catéchisme, le récit de la création de la femme à partir d'une côte de l'homme (III 101 : Deiws Rikijs billa : Sta ast ni labban, kai stas smunents ains ast « Dieu dit : il n'est pas bon que l'homme reste seul»= all. Gott der Herr sprach : Es ist nicht gut, das der Mensch allein sey, etc.). À ce propos, l'influence de l'allemand est patente, notamment sur l'ordre des mots (verbe à position finale dans les subordonnées, ex. verbe ast en fin de subordonnée comme all. sey), sur la création d'un article défini (stas smunents = all. der Mensch), sur la création d'un sujet vide dans les propositions impersonnelles (sta ast ni labban = all. es ist nicht gut), etc. De nombreuses particularités du texte prussien ont été évoquées pendant ces séances de lecture de texte, par exemple la question des formations de prétérit en prussien. 